\title{
APPLICATION OF COMPUTER NETWORK VIRTUAL INSTRUMENT TECHNOLOGY IN THE DEVELOPMENT OF A CONSTRUCTION MACHINERY REMOTE DETECTION SYSTEM
}

\author{
Haifeng Ma ${ }^{1 *}$, Qing Zhou ${ }^{2}$ \\ ${ }^{1}$ Qinhuangdao Vocational and Technical College, Qinhuangdao, 066100, China; \\ ${ }^{2}$ Shandong Vocational University of Foreign Affairs, Jinan 250100, China. \\ ${ }^{*}$ Corresponding author: Haifeng $\mathrm{Ma}$ \\ Email: mahaifengahd@126.com
}

\begin{abstract}
In order to meet the needs of remote vibration monitoring of construction machinery, data acquisition means, signal processing and analysis technology and Data Socket technology of computer network virtual instrument technology are applied to build a remote vibration monitoring and analysis system of construction machinery. First, according to the requirements of remote data acquisition system, the data acquisition terminal hardware, remote data transmission hardware and remote data transmission related servers in the monitoring and analysis system are selected and configured accordingly; moreover, the remote data transmission system, remote data receiving system and data management system are designed. then, Data Socket remote detection system and Web remote detection system are used to complete the requirements of remote detection of construction machinery device. Finally, the database and Lab-VIEW (virtual instrument) software are used. According to the system requirements and the software characteristics, the setting of login permissions and the related functions of the data management system are realized. After the development of the system, data simulation, laboratory test and onsite test are carried out. The experimental results show that the experimental data can be transmitted stably and correctly, and the system can realize real-time monitoring of construction machinery equipment, which verifies the feasibility of the constructed system. The mechanical vibration condition monitoring and analysis system based on networked virtual instrument not only integrates data acquisition, analysis and display, but also solves the complex problems in the development of general networked remote detection system, and improves the reliability and realtime of network communication. The research results provide a new method to solve the problem of remote mechanical vibration condition monitoring.
\end{abstract}

Keywords: Construction Machinery; Remote Detection; Virtual Instrument Technology; Lab-VIEW.

\section{Introduction}

In the daily work of the field of construction machinery, the staff often have to test and analyze the mechanical performance and parameters. The traditional test method is often completed by using large and expensive instruments. In addition, the compatibility between the traditional engineering test systems is very poor, and it is difficult to easily compatible with the relevant software and hardware resources, which increases the cost of testing and reduces the accuracy of test data. However, the structure of modern construction machinery is more complex, involving many technical fields, and there are many kinds of faults. Mechanical vibration is a common phenomenon in the working process of all kinds of machinery, which is also an important reason for reducing the safety and life of machinery operation [1]. Building a perfect and reasonable mechanical vibration detection system is of great engineering significance for analyzing the running state of machinery, predicting the potential safety hazards, and ensuring the safe and continuous operation of mechanical equipment. However, for the actual situation of China, in many applications, the on-site single machine closed detection system is difficult to meet the actual needs [2]. Therefore, the network-based remote multi-purpose monitoring and diagnosis system with the functions of real-time vibration signal acquisition and online spectrum analysis has attracted more and more attention of researchers. Rani and Sivia (2020) [3] developed a method of fractal antenna array virtual fault diagnosis instrument by using Lab-VIEW software. First, the fault of the antenna array was considered according to the radiation pattern. Then, an artificial 
neural network was established to fit the data points and minimize the mean square error by using $\mathrm{L}$ algorithm in MATLAB software. Finally, the neural network model was embedded into the virtual instrument, and the test mode was taken as the input to generate output for several faults in the antenna array in the proposed virtual instrument system.

Finally, the simulation and experimental results of fractal antenna array were verified. Bernardino et al. (2020) [4] studied a virtual instrument. The instrument could classify vehicles automatically and quasi instantaneously according to their categories when they were driving along the road. Vehicle classification was based on the accurate measurement of vehicle speed and wheelbase. Its research focus was to achieve accurate speed and wheelbase measurement, and then determine the type of vehicle through the development of software.

A new method of vehicle classification based on piezoelectric sensors was proposed in this system. Compared with the existing alternative systems (based on inductive loop, camera, optical fiber sensor or laser system), the system has important advantages, such as simple installation, non-invasive, and high classification success rate of more than 90\%. Singh and Malarvili (2020) [5] proposed a realtime electrocardiograph (ECG) monitoring system based on virtual instrument. The equipment was designed with surface electrode, lead, instrument amplifier and cut-off frequency passive low-pass filter. Then, by initializing the analog input and sampling rate, the ECG signal was transmitted to Lab-VIEW through data acquisition (DAQ) card for further analysis.

In addition, digital notch filter, band-pass filter and finite impulse response (FIR) high pass filter were used to remove the interference of power line. This finding provides a potential outcome for assessing cardiovascular disease. In the future, the equipment will perform standardized functional tests in healthy people, and extract significant features from ECG waveforms for cardiovascular disease analysis. In order to create an accurate displacement measurement method with low cost, strong anti-interference ability and stable performance in a wide range and nanometer level, $\mathrm{Xu}$ et al. (2019) [6] studied a nano time grating sensor based on AC electric field coupling. The LabVIEW software of virtual instrument development platform was combined with the hardware of PXI5422 arbitrary waveform generator of NI company, which could realize the frequency, amplitude, phase and other setting functions of standard waveform.

The experimental results show that the phase adjustment can improve the accuracy by adjusting the amplitude of the excitation signal, and avoid the influence of different installation positions on the nano time grating. Estrada et al. (2020) [7] found that railway track inspection is a necessary condition to ensure the safe operation of the track. Although the railway department has invested a huge amount of cost, time and manpower to ensure the performance and safety of the railway, due to the negligence of human inspection, accidents often occur throughout the year. Therefore, a simple method of railway track anomaly detection using visual perception and image processing technology was proposed. The system adopted the visual perception algorithm based on edge detection and feature extraction developed by LabVIEW. The algorithm could continuously record the track image, evaluate and detect the railway components, such as whether the loose bolts were loose and bent, and whether there were small cracks on the rail surface. The performance of the system was studied in the laboratory.

The results show that the system has good performance in railway track anomaly detection. Lazarevic et al. (2019) [8] pointed out that the main components of central heating substation system were heat exchanger and three-port valve. In order to improve the control technology and optimization algorithm of central heating substation system, the difficulty lies in how to apply the improved algorithm to the actual system. In order to solve this problem, a mathematical model was proposed. In this model, the central heating substation was decomposed into a simple model of its main components, and the influence of various parameters on the model performance was analyzed through numerical simulation. Lab-VIEW software package was used to develop real-time model. Moreover, on the embedded controller, the real-time processor was deployed as a three-layer application to realize data acquisition and communication with the programmable controller. Joseph and Modaran (2019) [9] learned that reverse osmosis process had become a prominent membrane-based desalination technology to meet the demand for fresh water.

The simulation results of reverse osmosis desalination process based on simplified function decomposition method were presented. Through the comparison with the experimental data of industrial seawater desalination, the effectiveness of the simulation model was verified. The author's work was carried out by using the control design and simulation toolkit in the Lab-VIEW 2011 environment. Simulation results show that maintaining a high feed temperature can reduce the specific energy consumption of reverse osmosis system under different fractionation recovery rates.

With the continuous development of Internet technology and remote detection technology of construction machinery, it was the development direction of mechanical equipment detection to realize remote detection of construction machinery with network technology as the carrier and mechanical fault detection technology. Therefore, a remote monitoring and analysis system for construction machinery vibration was proposed. The system could collect and analyze the vibration signal of construction machinery in real time, and could also use the network to monitor the vibration parameters of mechanical vibration in the working site continuously. 


\section{Method}

\subsection{Measurement and Control Technology and Virtual Instrument}

Today, measurement and control technology are not only an important pillar of industrial technology, but also a powerful guarantee to liberate the productive forces. In the field of cutting-edge science and technology, the realization of major achievements cannot be separated from the support of measurement and control technology. As a hot spot in the field of measurement and control, virtual instrument technology is widely used in all walks of life.

Meanwhile, it is more and more widely used in the field of mechanical equipment fault detection. Virtual instrument technology consists of computer, application software and instrument hardware. Software is the combination of hardware and computer, which connects other parts together, and then combines the computing ability of computer, the test of instrument hardware and the communication ability. The test data can be analyzed, processed, displayed and saved by master computer software programming. In the whole testing process, the display, analysis and other functions of the master computer are realized by programming software. It updates the way of traditional instrument measurement technology, and integrates the whole process of traditional instrument measurement in the visual software analysis platform, which makes the analysis more vivid.

\subsection{Network Virtual Instrument Technology and its Remote Data Transmission Mode}

Networked virtual instrument is a new instrument technology with the development of computer technology. It integrates network technology, computer technology, testing technology and communication technology, and is used to deal with remote data transmission and fault diagnosis. Different from the traditional test instrument, network virtualization instrument technology realizes network connection between test equipment and control equipment through computer. Test equipment can realize information exchange, remote control or monitoring with other computer resources on the network $[10,11]$. In addition, the network virtualization instrument technology concentrates the data acquisition process, data analysis process and data display process on the computer $[12,13]$. The processing process is to use the previously written software to process the data, that is, connecting the virtual instrument to the network is to put the compiled software into the network. The simplest way is to combine the test computer with the network card, and then directly connect to the network to realize the network connection of the test instrument $[14,15]$. Figure 1 shows the basic hardware structure of networked virtual instrument, which can be roughly divided into acquisition card, work host and network interface card.

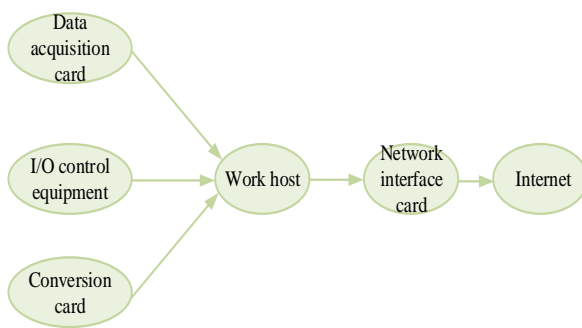

Figure 1 Basic hardware structure of Networked Virtual Instrument

In the basic structure of hardware shown in Figure 1, the front end of the whole test system is the acquisition card.

The signal acquisition hardware of the test system is composed of acquisition card and sensor. Its function is to convert the analogue signal input by the sensor into the digital signal that can be recognized by the computer, in order to facilitate the subsequent analysis work of the host [16]. The host computer will collect the digital signals transmitted by hardware for display, analysis, storage, simple evaluation and alarm operation. As the interface of host and network communication, the main function of network interface is to connect the host to the network to realize the networking of test instruments. Meanwhile, test resources and analysis resources are shared and provided to researchers and analysts for use and analysis, so as to realize "network is instrument" [17]. A complete system design, in addition to the hardware part, should also include the software part. Good software design can better play the function of hardware.

The design of network virtualization instrument also follows this principle. Figure 2 shows the structure of networked virtual instrument software, which is mainly composed of system management software, application program, instrument driver software and interface software $[18,19]$.

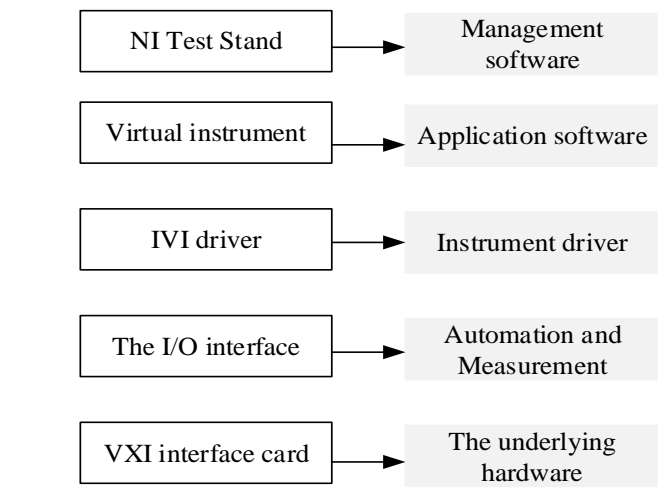

Figure 2 Software structure of Networked Virtual Instrument 
Figure 2 reveals that the bottom of the system is the system hardware component, which is directly connected with the test equipment to achieve data testing. The upper layer of the system hardware is the input/output (I/O) interface layer. When the test host and the test hardware are connected, the I/O interface is used to connect and realize the communication between the hardware and the host. The I/O interface is the channel between the two communications. The upper layer of I/O interface is IVI driver (a kind of driver in Lab-VIEW). The instrument driver is not available in the whole process of software communication. The host needs a certain driver software to complete the communication with the hardware through the I/O port. It does not communicate as soon as it is powered on. The main function of instrument driver software is to realize the communication and control between driver host and hardware. Finally, the system management software is located at the top of the software structure, and its function is to show the human-computer interaction interface, realize network connection, data report, and application database $[20,21]$.

The basic data transmission modes of networked virtual instrument can be divided into three types: 1) Client-Server (C/S) transmission mode; 2) Browser/Server (B/S) transmission mode; 3) mixed transmission mode of $\mathrm{C} / \mathrm{S}$ transmission mode and $\mathrm{B} / \mathrm{S}$ transmission mode. The $\mathrm{C} / \mathrm{S}$ transmission mode has the advantages of fast data transmission, beautiful operation interface and large amount of data transmission, but it also has the disadvantages of complex update software and poor compatibility.
It is generally used in local area network or has special requirements for data transmission, as shown in Figure 3. For B/S transmission mode, users can use the browser to detect the equipment's distance, without installing lots of applications like $\mathrm{C} / \mathrm{S}$. The advantage of this method is that it is easy to operate, easy to maintain, and can realize multi-user detection at the same time. Its disadvantage lies in its small amount of data transmission, low response speed, greatly affected by the speed of the network. The main applications based on $\mathrm{B} / \mathrm{S}$ mode architecture are web publishing technology and remote front panel control technology. The purpose of hybrid transmission mode of $\mathrm{C} / \mathrm{S}$ transmission mode and $\mathrm{B} / \mathrm{S}$ transmission mode is to combine the advantages of the two network data transmission modes and better use them in the actual measurement and control system. A measurement and control system can synthesize the parts with large amount of data interaction and small test scope into a system based on $\mathrm{C} / \mathrm{S}$ architecture, so as to effectively exchange test data in real time and detect multiple devices. On the contrary, for the devices with small amount of data, poor interactivity and wide monitoring range, $\mathrm{B} / \mathrm{S}$ mode can be adopted.

The real-time detection of browser client can be realized by using Web server. This exploration aims to study the construction machinery distance detection technology, in which the network virtual instrument technology is taken as a means of data transmission, which combines the Data Socket transmission technology with Web publishing technology, and is applied in the construction machinery remote detection system [22].

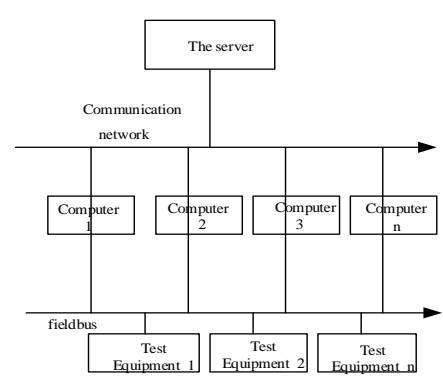

a)

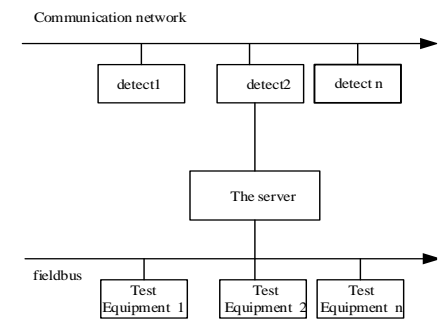

b)

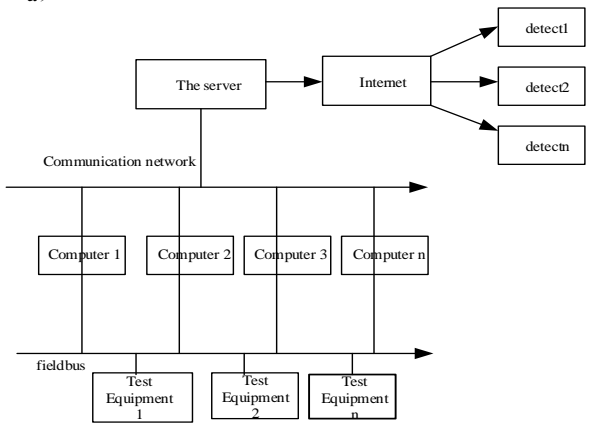

c)

Figure 3 Basic data transmission mode of networked virtual instrument (a) C/S transmission mode, b) $B / S$ transmission mode, $c$ ) mixed transmission mode) 


\subsection{Overall Structure of the System}

Figure 4 is the overall structure of the system proposed, which is composed of two parts, an on-site detection workstation and several remote detection and analysis centres. The main work of on-site detection workstation is data acquisition, signal processing, and monitoring.

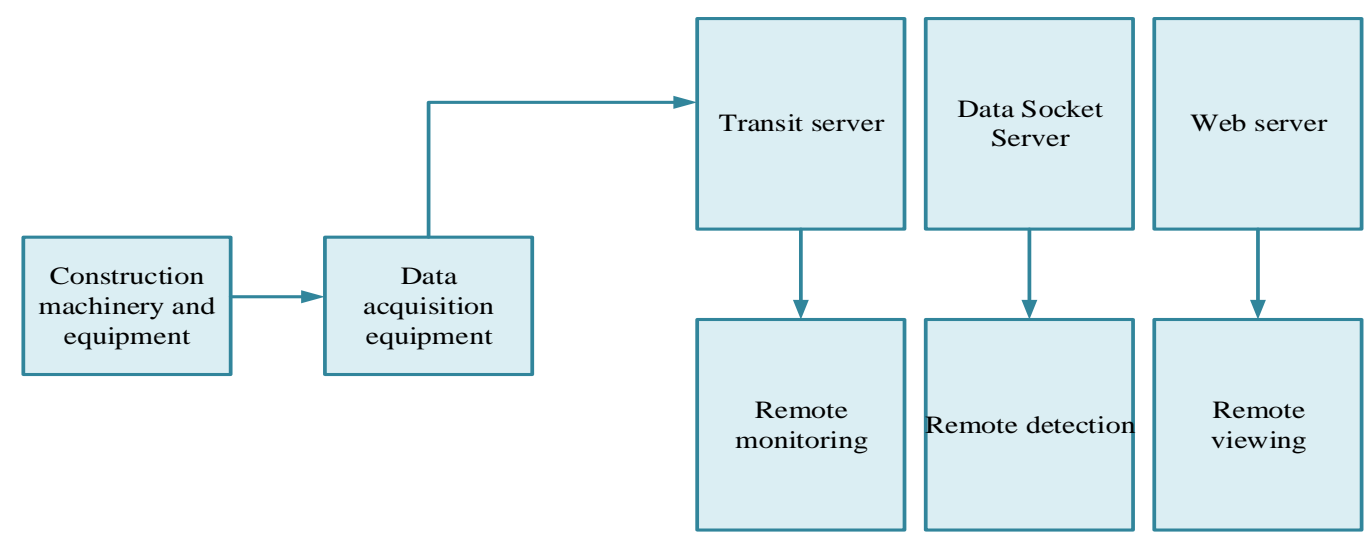

Figure 4: System structure diagram

Data Socket technology can be used to release the monitored data to the Internet.

The work content of the remote monitoring and analysis centre is to analyze and process the data collected by the detection workstation transmitted from the network in real time.

\subsection{Hardware Composition of the System}

The system proposed mainly monitors the vibration signal, uses the temperature information to evaluate the status of mechanical equipment, and detects whether the mechanical equipment has problems in real time. Common vibration sensors can be divided into vibration acceleration sensor, vibration velocity sensor and vibration displacement sensor. Because there are many kinds of construction machinery and a wide range of test frequency, vibration acceleration sensor is selected. At present, the most widely used vibration acceleration sensor in vibration measurement is piezoelectric vibration acceleration sensor (Figure 5) [23].

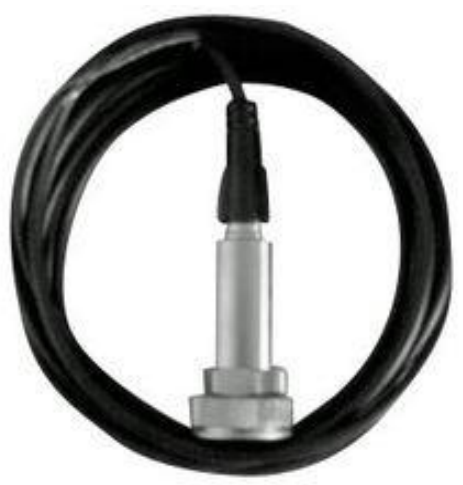

Figure 5: Piezoelectric vibration acceleration sensor

The main advantages of piezoelectric sensor are wide frequency range, large dynamic range, small mass, small volume, light weight and high sensitivity. As the A/D conversion card of on-site data acquisition will have a great impact on the real-time performance and accuracy of the monitoring system, the high-speed A/D conversion card selected is the data acquisition card of PCI bus [24].

\subsection{System Development Software and Signal Transmission and Communication Mode}

As the virtual instrument development software Lab-VIEW has powerful functions of data acquisition, analysis and display, it is selected as the system development software of this exploration. In the system, the real-time and stable communication between the on-site monitoring workstation and the remote monitoring and analysis centre is very important for the whole system [25,26]. Data Socket technology highly encapsulates the bottom layer of the program, and greatly simplifies the application program on the same computer. Moreover, it constructs a high-performance programming interface to share and release data between test equipment and automation software through the real-time data exchange process on different computers connected through the network. Therefore, the system constructed adopts Data Socket communication mechanism, and uses it to realize network programming. In addition, the onsite monitoring workstation and remote monitoring and analysis centre using Data Socket communication mechanism work independently. The on-site monitoring workstation can send data at any time, while the remote monitoring and analysis centre can receive data at any time, and the sending and receiving data are controlled by its underlying $\mathrm{T}$ protocol. 


\subsection{System Design and on-site Debugging}

In the system proposed, the main work of the onsite monitoring workstation is to collect and preprocess the data, that is, the vibration acceleration signal collected by piezoelectric acceleration sensor, charge amplifier and data acquisition card is collected and analyzed in frequency domain.

Then, on-site monitoring workstation communicates with client program. The sine wave signal generated by the signal generator is used as the simulation signal of vibration acceleration signal collected from the site containing mechanical equipment. The data acquisition card of on-site monitoring workstation is used for data acquisition.

The waveform and spectrum of the collected signal collected can be displayed on the interface of the on-site monitoring workstation in real time. The task of the remote analysis and test centre is to communicate with the server program, that is, to receive the vibration acceleration signal sent by the on-site monitoring workstation through the network, and analyze and process the acceleration signal. In addition, the acceleration signal needs to be integrated to obtain the vibration velocity and displacement parameters.

The Butterworth digital filter is used in both the remote detection and analysis centre program and the on-site monitoring program to filter the noise and unnecessary frequency components of the signal. Generally, the cut-off frequency and order of the filter are selected according to the test.
In addition, in order to reduce the periodic systematic error in the monitoring system, a Detrend VI (proper term) is constructed to eliminate the trend term. Detrend VI uses the Linear Fit VI module of ordinary least squares, which is to fit straight lines, to fit the trend term, and then subtract the trend term from the data.

\section{Results}

\subsection{Test of User Management System and Login Interface}

Figure 6 is the program block diagram of the front panel of the designed user management system and the front panel of the login system.

For the sake of system security, only one senior administrator is set in the user management system. The senior administrator has the operation authority to all registered users of the system. The administrator has lower authority than the senior administrator, who can only operate on the user and himself. The authority of user is lower than the administrator, and they can only modify their own password. In the designed login system, ordinary users only have three chances to enter the password each time they log in. If the password is entered incorrectly three times, the system will exit the login system. In the whole login system, if the user has wrong operation, the corresponding prompt window will pop up. On the contrary, if the user logs in successfully, the system will store the relevant information of the user.

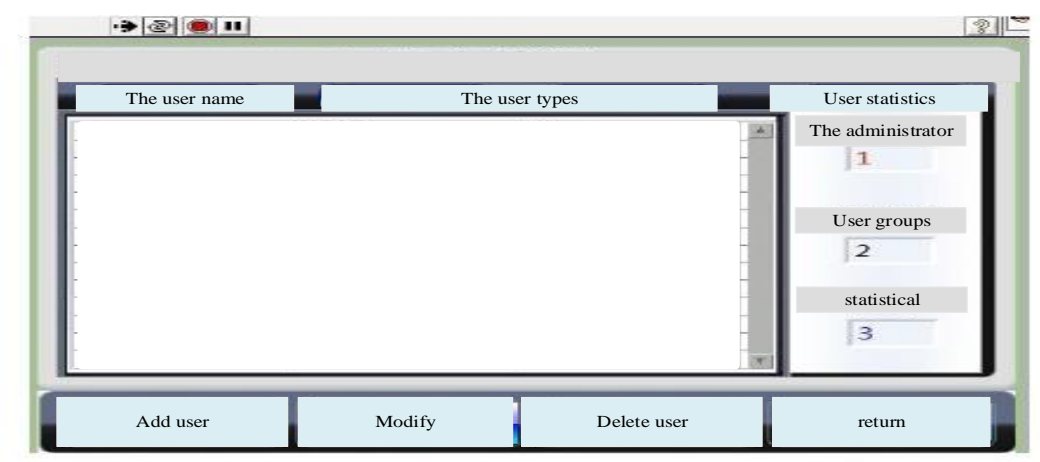

a)

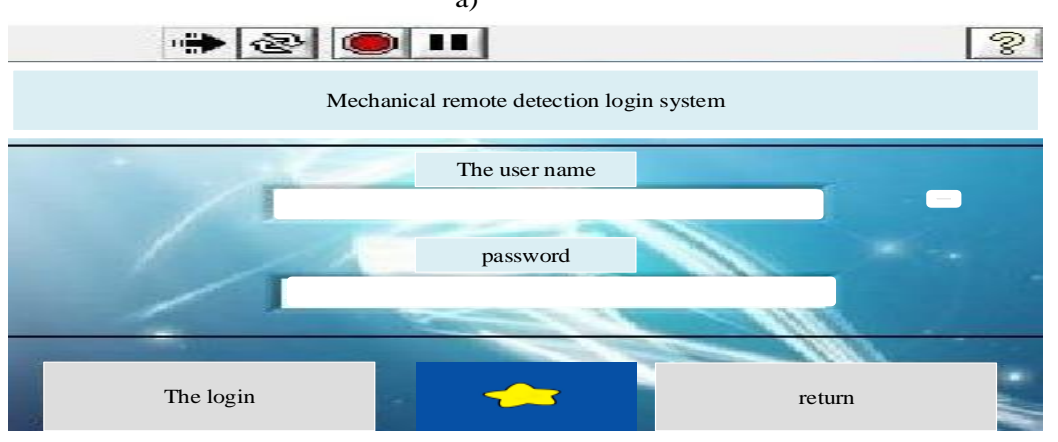

b)

Figure 6: Block diagram of user management system and login system (a) user management system diagram, b) login system diagram) 


\subsection{Remote Detection System Test}

Figure 7 shows the Data Socket remote data transmission test diagram and Web remote release test diagram.

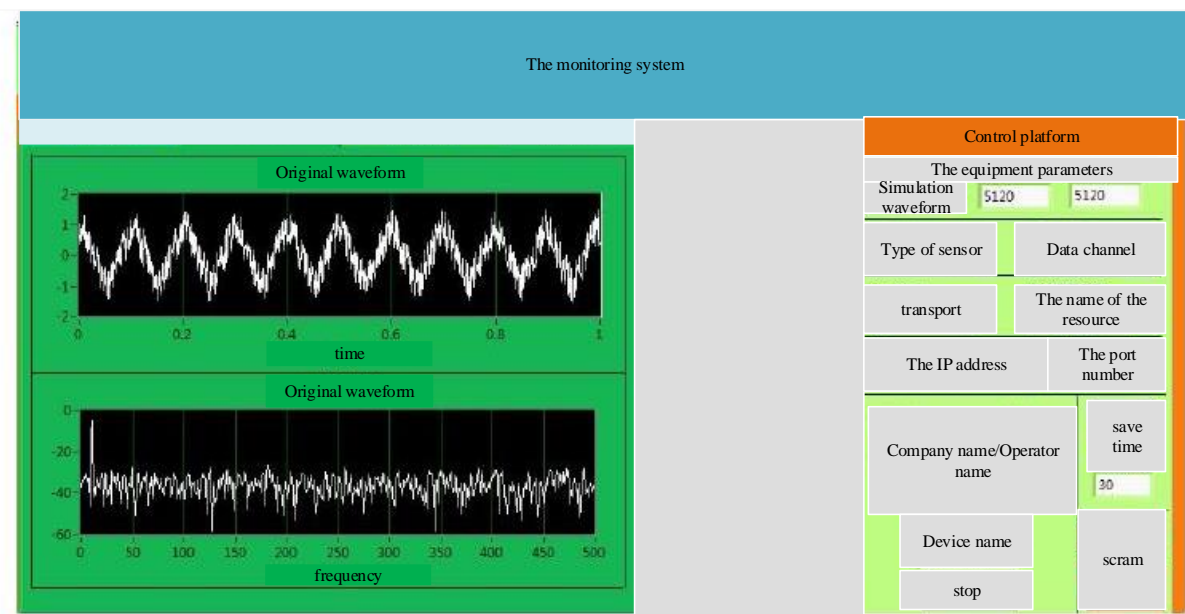

a)
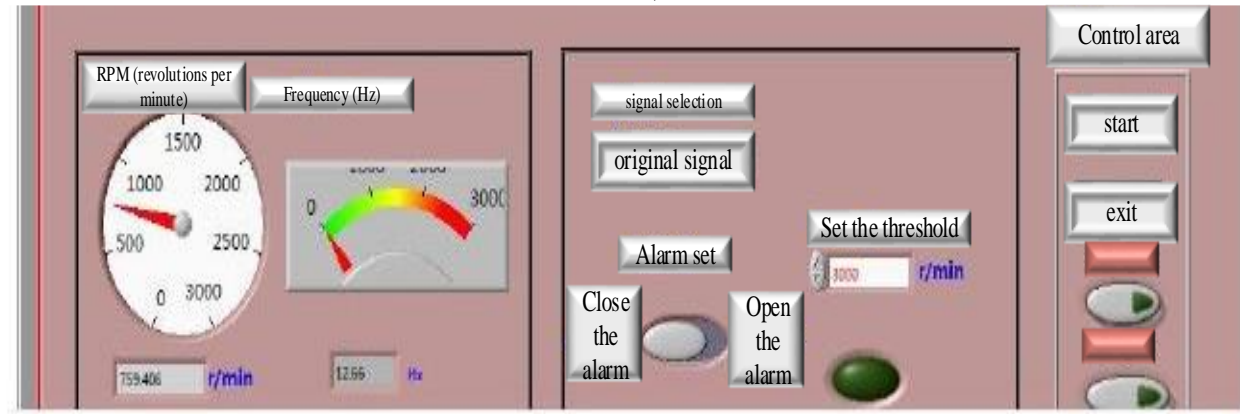

b)

Figure 7: Data Socket remote data transmission test diagram and Web remote release test diagram a) Data Socket remote data transmission test diagram, b) Web remote release test diagram)

Figure 7 reveals that Data Socket supports a variety of data transmission, such as string, array, and value. In the data transmission of construction machinery remote detection system, the data is packaged into clusters for transmission, which avoids the problem that the data received by the receiver cannot be restored due to the lack of relevant parameters. For Web remote publishing, a server can support multiple computers to access at the same time, which also means that different browser customers can access the application in the different place and at different times, which is convenient for staff in different regions to study the same equipment.

\subsection{Data Storage and Management System Test}

Figure 8 shows the GPRS receiver data management test diagram.

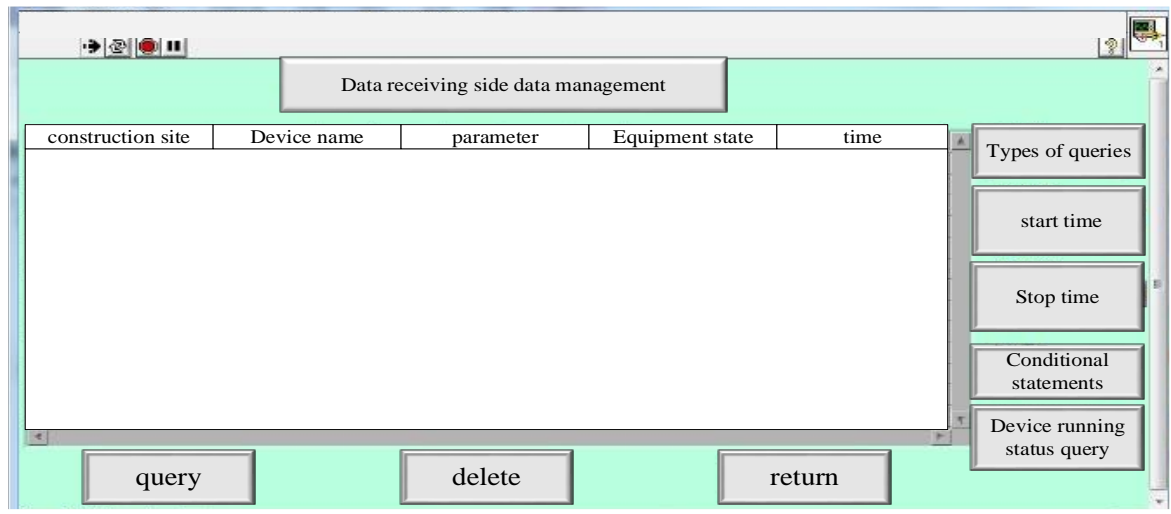

Figure 8: Test diagram of GPRS receiver data management 
Figure 8 reveals that the function of deleting the data in the database is to index the primary key content when the mouse arrow locates to a row of data in the table, add it to the SQL statement to complete the SQL deletion statement, and add it to the deletion control of Lab-VIEW to delete the content.

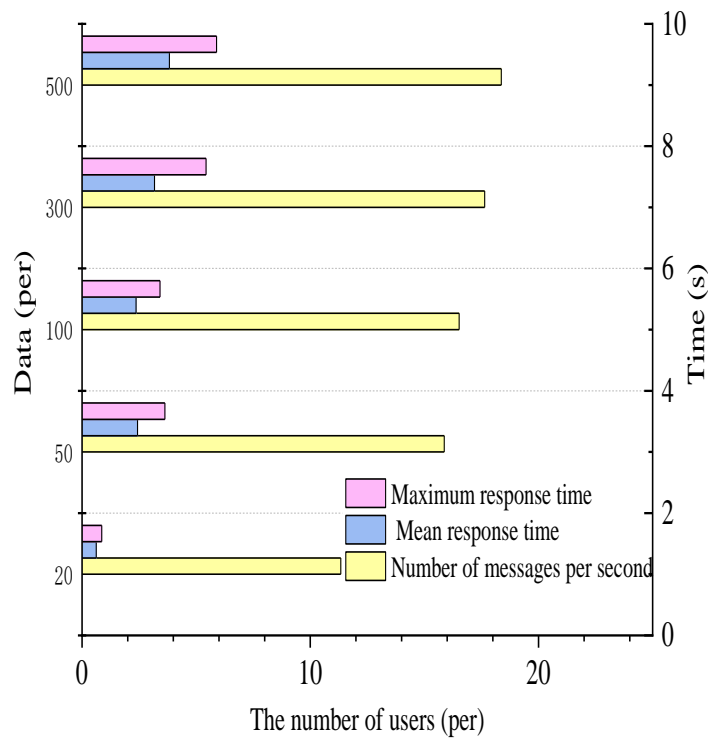

a) performance analysis of general system Figure 9: Performance analysis in simple business

\subsection{Simple Fault Test}

The business process of multiple users in dealing with simple mechanical equipment failure is simulated. The number of selected users is $2,50,10$, 15 and 17. Several continuous tests are carried out respectively. Figure 9 shows the results.

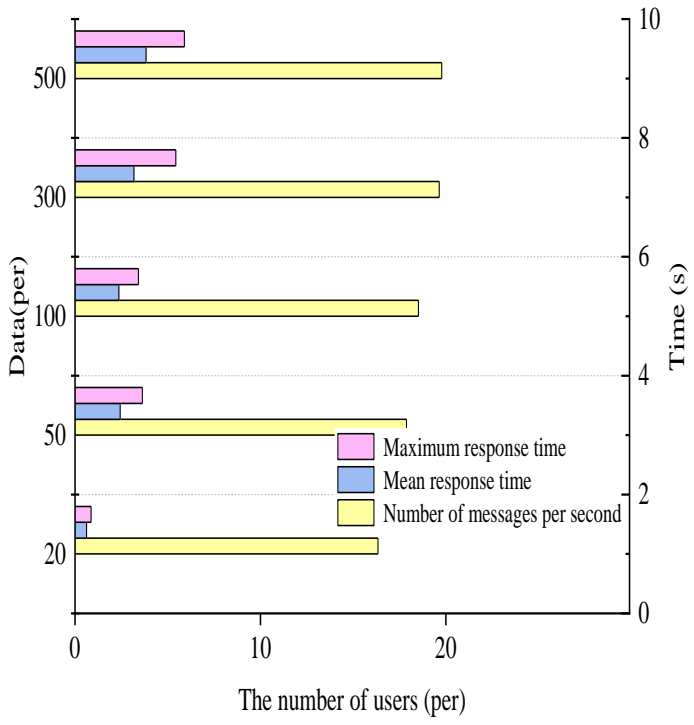

b) performance analysis of the proposed system

data acquisition terminal and data transmission terminal is completed, and the configuration of remote data transmission server is completed to provide corresponding support for the system. Then, the basic knowledge of remote data transmission is introduced in detail, and the basic mode of remote data transmission is determined. By using Data Socket and Web publishing, the requirement of remote detection of mechanical equipment is basically realized, and it is verified by data simulation and on-site test.

Finally, the login permission setting, data management system, printing reports, waveform display and other related functions are realized, which improves the interaction of the system. Compared with other remote detection technologies of construction machinery, the proposed system is stabler and more reliable in the real-time transmission of remote data, and provides a stable communication foundation for the remote detection system of construction machinery.

Compared with the previous system, the proposed system can save data information more accurately and vividly, which provides convenience for staff to analyze and view data. 


\section{Conclusion}

Remote data transmission technology and construction machinery state detection technology are studied, and the two technologies are combined to realize the remote detection of construction machinery. In the whole system construction process, Data Socket remote data transmission technology and Web publishing technology are focused on. In addition, the application of vibration signal processing in construction machinery detection is also studied. Finally, the communication between the on-site data and the distance detection terminal is realized by using Lab-VIEW software.

Although the remote detection function of construction machinery is basically realized, there are still some shortcomings and deficiencies in the system. In the problem of vibration signal processing and analysis, it is necessary to analyze the vibration signal more accurately.

Especially in the remote detection, it is necessary to add the evaluation standard of equipment operation status, in order to reduce the occurrence of false alarm. These problems are also the direction of follow-up research.

\section{References}

[1] Patrick R., Nathalie W. (2020) Virtual Reality als Instrument zur Gewinnung von Nutzerfeedback zu Technologieszenarien am Beispiel urbaner Mobilitt. HMD Praxis der Wirtschaftsinformatik, 57(2), 230-243.

[2] Su L. (2019) Design of Optical Fiber Displacement Measurement System Based on Virtual Instrument. International Journal of Social Science and Education Research, 2(6), 159-163.

[3] Rani S., Sivia J. S. (2020) Design and development of virtual instrument for fault diagnosis in fractal antenna array. International journal of RF and microwave computer-aided engineering, 30(1), e22026.1-e22026.10.

[4] Bernardino G., Francisco J. J., José D. Fr. (2020) A Virtual Instrument for Road Vehicle Classification Based on Piezoelectric Transducers. Sensors, 20(16), 4597.

[5] Singh O. P., Malarvili M. B. (2020) Virtual Instrument Based Real Time ECG Monitoring Device. IOP Conference Series: Materials Science and Engineering, 884(1), 012011.

[6] Xu L., Xia Y., Jun Z, et al. (2019) Experimental Research of Displacement Sensor Based on Virtual Instrument Technology. Journal of Physics Conference Series, 1168(3), 032139.

[7] Estrada L., Arau J., Sciubba E. (2020) Real-Time Hardware in the Loop Simulation Methodology for Power Converters Using LabVIEW FPGA. Energies, 13(2), 373.
[8] Lazarevic S., Congradac V., Andelkovic A. S, et al. (2019) A novel approach to real-time modelling of the district heating substation system using LabVIEW. Journal of Cleaner Production, 217(APR.20), 360-370.

[9] Joseph A., Modaran V. (2019) Dynamic simulation of the reverse osmosis process for seawater using LabVIEW and an analysis of the process performance. Computers \& Chemical Engineering, 121(FEB.2), 294-305.

[10] Xiao Q., Wang L., Fang J, et al. (2020) Research on the application of virtual instrument technology in simulation training. MATEC Web of Conferences, 309, 03022.

[11] Schreurs B., Beemt A. V. D., Moolenaar N, et al. (2019) Networked individualism and learning in organizations: An ego-network perspective on informal learning ties. Journal of Workplace Learning, 31(2), 95-115.

[12] Thirunavukkarasu S., Arjun V., Rao B. P. C, et al. (2019) Development of a High-Sensitive Dual Frequency Remote Field Eddy Current Instrument for Inspection of Ferromagnetic Steam Generator Tubes. IETE Technical Review, 36(2), 203-208.

[13] Xu Z., Lin Z., Xiao Q. (2019) A remote field eddy current probe for outside inspection of pipe defects. International Journal of Applied Electromagnetics and Mechanics, 59(4), 15431551.

[14] Zhao J., Li X. L., Ma H. F. (2020) The Application of BP Neural Network Algorithm in Rapid Design of the Vertical Boom of Bridge Inspection Vehicle. Journal of Physics: Conference Series, 1570(1), 012005.

[15] Xu Z., Lin Z., Xiao Q. A remote field eddy current probe for outside inspection of pipe defects. International Journal of Applied Electromagnetics and Mechanics, 59(4), 1543-1551.

[16] Kamaeva S. S., Kolesnikov I. S., Eremin N. A, et al. (2019) Remote inspection by the magnetic tomography method (MTM) to prevent the risks imposed by exploitation of Arctic offshore pipelines. IOP Conference Series: Materials Science and Engineering, 700(1), 012051.

[17] Thirunavukkarasu S., Arjun V., Rao B. P. C, et al. (2019) Development of a High-Sensitive Dual Frequency Remote Field Eddy Current Instrument for Inspection of Ferromagnetic Steam Generator Tubes. IETE Technical Review, 36(2), 203-208.

[18] Tan Y., Li S., Wang Q. (2020) Automated Geometric Quality Inspection of Prefabricated Housing Units Using BIM and LiDAR. Remote Sensing, 12(15), 2492.

[19] Kamaeva S. S., Kolesnikov I. S., Eremin N. A, et al (2020). Remote inspection by the magnetic tomography method (MTM) to prevent the risks imposed by exploitation of Arctic offshore 
pipelines. IOP Conference Series: Materials Science and Engineering, 700(1), 012051.

[20] Zhang D., Watson R., Dobie G, et al. (2020) Quantifying impacts on remote photogrammetric inspection using unmanned aerial vehicles. Engineering Structures, 209, 109940.

[21] Zinkevich I., Philipp M., Skvortsova O, et al. (2019) Innovative methods of concrete dams' inspection. IOP Conference Series Earth and Environmental Science, 403, 012240.

[22] Li L., Xia Z., Yang Y, et al. (2020) Atomistic simulations on nanoimprinting of copper by aligned carbon nanotube arrays under a highfrequency mechanical vibration. Nanotechnology, 31(4), 045303.

[23] Li X., Xue L., Chen Z., et al. (2020) Analysis method for magneto-mechanical coupled vibration of the in-vessel structures considering halo current effect and application to HL-2M tokamak. Fusion Engineering and Design, 156, 111612.
[24] Fernando I., Fei J., Stanley R. (2019) Measurement and analysis of vibration and mechanical damage to bananas during longdistance interstate transport by multi-trailer road trains [J]. Postharvest Biology and Technology, 158, 110977.

[25] Andrés F. Ríos-Mesa., Gallego R. Z., Osorio M, et al. (2020) Effect of vehicle vibration on the mechanical and sensory properties of avocado ( Persea Americana Mill. Cv. Hass) during road transportation[J]. International Journal of Fruit Science, 20(146), 1-16.

[26] Wang G., Lv B., Liu L, et al. (2020) Influence of vibration on mechanical polishing microstructured surface using discrete element method[J]. International Journal of Machining and Machinability of Materials, 22(1), 24. 